Original article

\title{
COMPARATIVE EVALUATION OF SOMATIC PROTEINS OF THE NYMPHAL STAGE OF LINGUATULA SERRATA ISOLATED FROM GOATS AND CATTLE IN NORTHWEST IRAN
}

\author{
N. HAJIPOUR ${ }^{1}$, M. TAVASSOLI ${ }^{1}$, H. TAYEFI-NASRABADI $^{2}$ \\ $\&$ SH. SEYEDI ${ }^{3}$ \\ ${ }^{1}$ Department of Pathobiology, Faculty of Veterinary Medicine, Urmia University, \\ Urmia, Iran; ${ }^{2}$ Department of Basic Sciences, Faculty of Veterinary Medicine, \\ Tabriz University, Tabriz, Iran; ${ }^{3}$ Department of Immunology, Faculty of Medicine, \\ Urmia University of Medical Sciences, Urmia, Iran
}

\section{Summary}

Hajipour, N., M. Tavassoli, H. Tayefi-Nasrabadi \& Sh. Seyedi, 2016. Comparative evaluation of somatic proteins of the nymphal stage of Linguatula serrata isolated from goats and cattle in Northwest Iran. Bulg. J. Vet. Med.,19, No 3, 242-246.

Because the nymphal stages of $L$. serrata isolated from different animals have different strains, it was assumed that their somatic proteins would be different. The present study was conducted to evaluate and characterise the somatic proteins of $L$. serrata nymphs, isolated from goats and cattle mesenteric lymph nodes. Nymphs were collected from the infected mesenteric lymph nodes of slaughtered cattle and goats. Somatic proteins were prepared by homogenising nymphs and then were electrophoresed using SDS-PAGE. Following SDS-PAGE, somatic proteins of the nymphal stage of L. serrata in our samples were characterised by the presence of 6 protein bands with molecular weights of 14.4, 32, 36, 48,75 and $100 \mathrm{kDa}$ in goats and 7 protein bands with molecular weights of $14.4,32,36,48,75,100$ and $120 \mathrm{kDa}$ in cattle. The evaluation of somatic proteins showed slight differences in the mesenteric lymph nodes of goats and cattle. The findings of this study can be used both for further evaluation of their antigenicity for diagnostic purposes and for development of protective vaccines.

Key words: cattle, goat, Linguatula serrata, SDS-PAGE, somatic proteins

\section{INTRODUCTION}

As one of the parasitic zoonoses, Linguatula serrata inhabits the canine respiratory system as its final habitat. Discharged eggs from the definitive host's nasopharyngeal secretion are ingested by the grazing herbivores where they hatch into larvae which migrate into the mesenteric lymph nodes, liver and lungs, where eventually they develop into infective nymphs (Rezaei et al., 2011). The pathogenesis of linguatulosis is not clear, but it has been shown that proteolytic enzymes may have a role in it (Alcala-Canto et al., 2007). According to Yagi et al. (1996) and 
Siavoshi et al. (2002), linguatulosis in men can be caused by either the egg (visceral form) or nymph stage of the parasite (nasopharyngeal form). Nasopharyngeal linguatulosis, known as Halzoun or Marrara syndrome, often occurs after consumption of raw or undercooked viscera (liver, lung and lymph nodes) of infected animals. The low mortality/high morbidity typical of helminth infections caused by gastrointestinal worms, schistosomes, nematodes or cestodes, presumably reduces the interest and thus focused investigation into their control and treatment. At the same time, however, these infections typically provoke severe disablement through pathological lesions and malnutrition. Thus a better understanding of the hostparasite inter phase in helminth infections is urgently required and, given the recent technical innovations in molecular biology and cellular immunology, quite feasible. The new era of recombinant antigens, $\mathrm{T}$ cell clones and anti-idiotypic reagents is ideally suited to the immediate objectives of reliable diagnosis, vaccine production and alleviation of immune pathological consequences. The mother technology in the field of molecular biology has caused new trend in the research which enables us to diagnose the infectious stages of parasites (Parkhouse \& Harrison. 1989). One of these methods is identifying the somatic proteins of the parasites by sodium dodecyl sulfate polyacrylamide gel electrophoresis (SDS-PAGE). Somatic proteins whose identification is essential for an early diagnosis of the parasitic infections, are considered as surface and secreted proteins are the most likely targets for protective immune responses (Pritchard, 1986). A thorough study of parasitic helminths somatic proteins is a pre-requisite for control programmes, which are intended to diminish pathologi- cal sequel and are based on accurate immunochemical diagnosis, protection by vaccination, and perhaps immune modulation (Parkhouse \& Harrison, 1989).

To date, the existing research has focused on the somatic proteins in isolated L. serrata nymph from mesenteric lymph nodes of goats (Youssefi et al., 2014). Therefore, the objective of this study was to compare the somatic proteins of the nymphal stage of $L$. serrata isolated from goats and cattle mesenteric lymph nodes.

\section{MATERIALS AND METHODS}

\section{Study area}

This study was conducted on slaughtered goats and cattle in Slaughterhouse of Tabriz, East Azerbaijan, in 2015. The city of Tabriz is located in the Northwest of Iran (geographical coordinates $38^{\circ} 4^{\prime} \mathrm{N}$ and $46^{\circ} 18^{\prime} \mathrm{E}$ ) with a human population of almost 1.5 million. The studied region has a moderate climate in winters and mild in summers, with an annual rainfall of about $388 \mathrm{~mm}$.

\section{Preparation of the samples}

The mesenteric lymph nodes from 130 goats (95 females and 35 males) and 80 cattle (60 male and 20 female) were examined for $L$. serrata nymphs between May and January 2015 after collection of mesenteric lymph nodes of goats and cattle slaughtered in Tabriz slaughterhouse. They were transferred to the Laboratory of veterinary parasitology, Faculty of Veterinary Medicine, Urmia University. Each lymph node was cut into small pieces and immersed in the Petri dish containing tepid water and left for 15 min to allow nymphs to come out from the tissue. Then, apparently normal lymph nodes were digested in $200 \mathrm{~mL}$ of digestion solution 
containing $5 \mathrm{~g}$ of pepsin and $25 \mathrm{~mL}$ hydrochloric acid in $1000 \mathrm{~mL}$ distilled water, and were incubated at $37{ }^{\circ} \mathrm{C}$ for $24 \mathrm{~h}$ (Razavi et al., 2004). Recovered nymphs were washed three times in phosphatebuffered saline (PBS, $\mathrm{pH}=7.2$; Sigma), sonicated in five cycles of $70 \mathrm{kHz}(30 \mathrm{~s}$ each) at $4{ }^{\circ} \mathrm{C}$ and centrifuged at $4,000 \mathrm{~g}$ for $15 \mathrm{~min}$. The supernatant was collected and filtered through $0.4 \mu \mathrm{m}$ sieves and stored at $-80{ }^{\circ} \mathrm{C}$ until analysis. The protein concentration was measured according to Bradford (1976).

Sodium dodecyl sulphate polyacrylamide gel electrophoresis (SDS-PAGE)

Somatic proteins were separated by SDSPAGE as described by Laemmli (Brant $e t$ al., 2010), using a mini protein II cell (Bio-Rad). The proteins were heated in a water bath at $100{ }^{\circ} \mathrm{C}$ for $10 \mathrm{~min}$. Then, they were added to each well of a $7 \%$ stacking gel and $12 \%$ separating gel. SDSPAGE was carried out at $120 \mathrm{~V}$, for $4 \mathrm{~h}$. Gels were stained with $0.05 \%$ Coomassie brilliant blue staining (G-250, Cat. No: B0770, Sigma Chemicals). The molecular weights of proteins were determined by comparing their migration distance against that of a known molecular marker. For size estimation in SDS-PAGE, a prestained protein marker at a range of 14.4$130 \mathrm{kDa}$ molecular weight (PS10 plus) was used.

\section{RESULTS}

Findings showed that 120 out of 130 $(92.3 \%)$ goats and 40 out of $80(50 \%)$ cattle were infected with nymph stages of L. serrata. The range, indicating the number of nymphs collected from each infected lymph node, varied from 50 to 400 for goats and 5-15 for cattle. The evaluation of the lanes containing somatic pro-

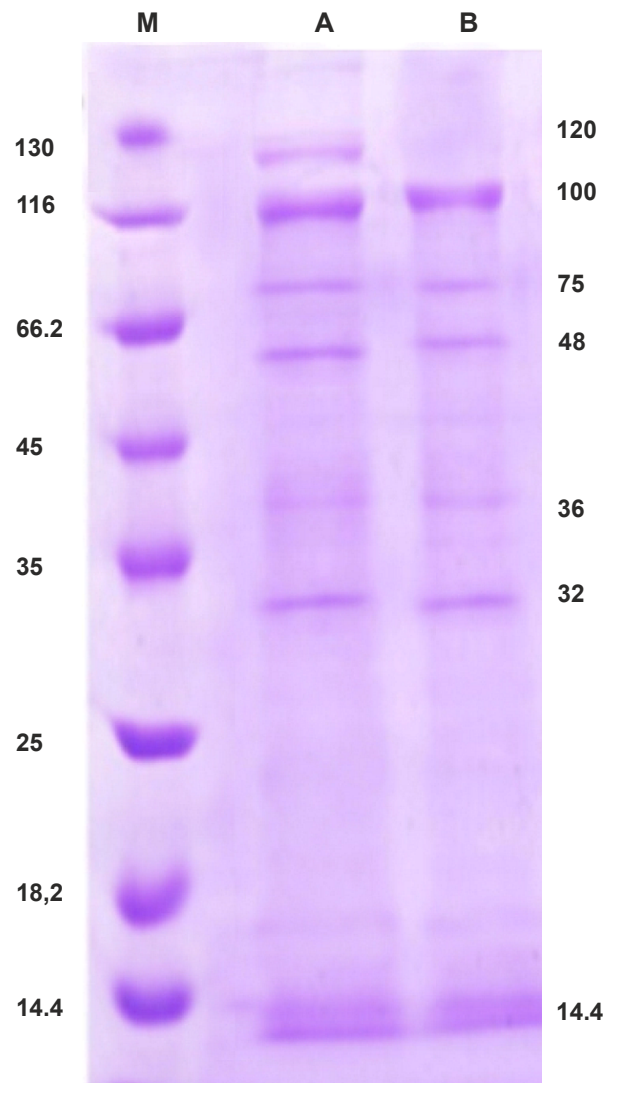

Fig. 1. SDS-PAGE pattern of somatic proteins in nymphal stage of Linguatula serrata. Lane A - cattle, Lane B - goat; Lane M - molecular weight marker $(\mathrm{kDa})$.

tein of L. serrata nymphs isolated from mesenteric lymph nodes of goats and cattle revealed six $(14.4,32,36,48,75$ and $100 \mathrm{kDa})$ and seven $(14.4,32,36,48,75$, 100 and $120 \mathrm{kDa}$ ) protein bands, respectively.

\section{DISCUSSION}

Linguatulosis is of a veterinary and public health importance in the world. As intermediate hosts, goats and cattle, like other ruminants, are essential in the life cycle of L. serrata. A close contact between dogs 
and the intermediate hosts plays an important role in transmission of L. serrata in our research context. Several studies have been conducted in Iran to determine the prevalence of L. serrata in ruminants. For instance, prevalence rates of $44 \%$ were recorded in cattle in Urmia slaughterhouse, Iran (Tajik et al., 2006). Moreover, a prevalence of $16.22 \%$ and $49.1 \%$ in mesenteric lymph nodes from cattle and goats respectively was reported in Kerman (Nourollahi Fard et al., 2010; 2012). In the present study, prevalence rates of $92.3 \%$ and $50 \%$ were observed in goat and cattle mesenteric lymph nodes respectively. High prevalence rate of infection in our research samples may be a manifestation of climatic parameters that enhance survival of parasite eggs in vegetables, fruits and water resources.

An important aspect in diagnosis of linguatulosis is the identification of antigens which are specific and highly immunogenic for the infected host. Since this parasite stimulates the host immune system and negatively influences many immune dominant antigens, parasitic infections occur in animals, resulting in diminished productivity. Therefore, an early diagnostics seems to be vital in preventing parasite growth. To aid the development of early diagnostics, information about somatic proteins of the nymph stage of $L$. serrata that may elicit a host's immune response, is required (Parkhouse \& Harrison, 1989). In the present study, somatic proteins of L. serrata nymphs isolated from goats and cattle mesenteric lymph nodes turned out to be different in their protein banding patterns on SDSPAGE. Our study showed 6 protein bands in goats $(14.4,32,36,48,75$ and 100 $\mathrm{kDa})$ and 7 protein bands in cattle (14.4, $32,36,48,75,100$ and $120 \mathrm{kDa}$ ). Out of these bands, the 36 and $48 \mathrm{kDa}$ ones were more prominent. Our study results are incompatible with the findings of Youssefi et al. (2014) who researched on electrophoretic pattern of somatic proteins of $L$. serrata nymph isolated from goat mesenteric lymph nodes and found out 6 protein bands $(19,20,36,48,75,100 \mathrm{kDa})$. However, our research scope and findings were different; we extended our focus from goat to cattle mesenteric lymph nodes and our results indicated 2 different bands of protein. Tavassoli et al. (2014) attributed this difference to the fact that the nymphal stage of $L$. serrata has different strains. In their study on $L$. serrata isolated from different farm animals of Iran, the authors showed molecular differences among them by amplifying and sequencing of $18 \mathrm{~S}$ rRNA of all L. serrata isolated from farm animals. Their results revealed that the lowest diversity of nucleotide sequences $(98.8 \%)$ belonged to sheep, cattle and dogs, while the highest sequence identity $(100 \%)$ was in camels and goats. Similarly, our findings can provide support for data of Jones \& Riley (1991) on frontal glands of a porocephalid pentastomid (Porocephalus crotali) in rats as intermediate hosts; in agreement with their investigation, our findings indicated the six protein bands in which the 14.4 $\mathrm{kDa}$ band was the same.

On the basis of our findings, we can determine the most important antigenic protein of $L$. serrata in order to design ELISA and relevant vaccines.

\section{ACKNOWLEDGMENTS}

This project was funded by the Urmia University, Urmia, West Azerbijan, Iran. The resultant part of this article is adopted from Ph.D thesis of Mr. Nasser Hajipour (Code Proposal: 1134667). We also thank Dr. Reza Habibian, Mr. Asghar Aliyari, Dr Mostafa Golabi and Mr Armen Badali for their technical support. 


\section{REFERENCES}

Alcala-Canto, Y., A. Alberti-Navarro \& F. Ibarra-Velarde, 2007. Serine protease activity demonstrated in the larval stage of the pentastomid Linguatula serrata. Parasitology Research, 100, 1011-1014.

Bradford, M. M., 1976. A rapid and sensitive method for the quantitation of microgram quantities of protein utilizing the principle of protein-dye binding. Analytical Biochemistry, 72, 248-254.

Brant, M. P. R., S. Guimarães, J. A. SouzaNeto, P. E. M. Ribolla \& T. C. G. Oliveira-Sequeira, 2010. Characterization of the excretory/secretory products of Dermatobia hominis larvae, the human bot fly. Veterinary Parasitology, 168, 304-311.

Jones, D. \& J. Riley, 1991. An ELISA for the detection of pentastomid infections in the rat. Parasitology, 103, 331-337.

Nourollahi Fard, S. R., R. Kheirandish, E. N. Asl \& S. Fathi, 2010. The prevalence of Linguatula serrata nymphs in goats slaughtered in Kerman slaughterhouse, Kerman, Iran. Veterinary Parasitology, 171, 176-178.

Nourollahi Fard, S. R., N. Ghalekhani, R. Kheirandish, S. Fathi \& E. Norouzi Asl, 2012. The prevalence of Linguatula serrata nymphs in camels slaughtered in Mashhad slaughterhouse, Northeast, Iran. Asian Pacific Journal of Tropical Biomedicine, 2, 885-888.

Parkhouse, R. M. \& L. J. Harrison, 1989. Antigens of parasitic helminths in diagnosis, protection and pathology. Parasitology, 99, Suppl, S5-S19.

Pritchard, D., 1986. Antigens of gastrointestinal nematodes. Transactions of the Royal Society of Tropical Medicine and Hygiene, 80, 728-734.

Razavi, S. M., S. S. Shekarforoush \& M. Izadi, 2004. Prevalence of Linguatula serrata nymphs in goats in Shiraz, Iran. Small Ruminant Research, 54, 213-217.

Rezaei, F., M. Tavassoli \& A. Mahmoudian, 2011. Prevalence of Linguatula serrata infection among dogs and domestic ruminants in North West of Iran. Veterinarni Medicina, 56, 561-567.

Siavoshi, M., M. Asmar \& A. Vatankhah, 2002. Nasopharyngeal pentastomiasis (Halzoun): Report of 3 cases. Iranian Journal of Medical Sciences, 27, 191-192.

Tajik, H., M. Tavassoli, B. Dalirnagadeh \& M. Danehloipour, 2006. Mesenteric lymph nodes infection with Linguatula serrata nymphs in cattle. Iranian Journal of Veterinary Research, 7, 82-85.

Tavassoli, M., S. A. Gorashi, S. Shamsi \& N. Hajipour, 2014. Molecular differences between Linguatula serrata isolated from differernt farm animals of Iran. In: Proceedings from the $22^{\text {nd }}$ Iranian Congress on Infectious Diseases and Tropical Medicine, Iran.

Yagi, H., S. el Bahari, H. A. Mohamed, el-R. S. Ahmed, B. Mustafa, M. Mahmoud, M. B. Saad, S. M. Sulaiman \& A. M. el Hassan, 1996. The Marrara syndrome: A hypersensitivity reaction of the upper respiratory tract and buccopharyngeal mucosa to nymphs of Linguatula serrata. Acta Tropica, 62, 127-134.

Youssefi, M., R. Tabaripour, A. Gerami \& V. Omrani, 2014. Electrophoretic pattern of Linguatula serrata larva isolated from goat mesenteric lymph node. Journal of Parasitic Diseases, DOI 10.1007/s12639014-0497-4.

Paper received 02.06.2015; accepted for publication 15.07.2015

\section{Correspondence:}

Mousa Tavassoli

Department of Pathobiology,

Faculty of Veterinary Medicine,

Urmia University, Urmia, Iran

e-mail: mtavassoli2000@yahoo.com 